\title{
Berührungslose Schallfeldmessung für den Einsatz in Flugzeugtriebwerken
}

\author{
Daniel Haufe, Andreas Fischer, Lars Büttner und Jürgen Czarske \\ Technische Universität Dresden, Fakultät Elektrotechnik und Informationstechnik; Professur für Mess- \\ und Prüftechnik; Helmholtzstr. 18, D-01069 Dresden \\ Tel.-Nr.: +49 351 463-33894; E-Mail-Adresse: daniel.haufe@tu-dresden.de
}

\section{Motivation}

Die Belastung der Bevölkerung durch Fluglärm weltweit zu reduzieren ist das erklärte Ziel namhafter Hersteller, Forschungseinrichtungen und Behörden im Bereich des Flugwesens [Eur01]. Inbesondere die Lärmentstehung und -reduzierung in Triebwerken ist daher Gegenstand aktueller Forschung. So soll beispielsweise ein besseres Verständnis aeroakustischer Phänomene in sogenannten Bias-Flow-Linern (siehe Abbildung 1) erlangt werden, die zur Dämpfung von Triebwerkslärm eingesetzt werden [Heu10a]. Aus den gewonnenen Erkenntnissen soll schließlich eine Minimierung des abgestrahlten Schalls erzielt werden. Zu diesem Zweck werden berührungslosen Messverfahren benötigt, die in einem solchen Liner neben der Strömungsgeschwindigkeit auch die sogenannte Schallschnelle rückwirkungsfrei erfassen können. Die Schallschnelle ist ein Geschwindigkeitsvektor, der die schallinduzierte Teilchenschwingung beschreibt. Die Kenntnis der Schallschnelle erlaubt gegenüber der skalaren akustischen Größe Schalldruck zusätzliche Aussagen zur lokalen Ausbreitungsrichtung des Schalls.

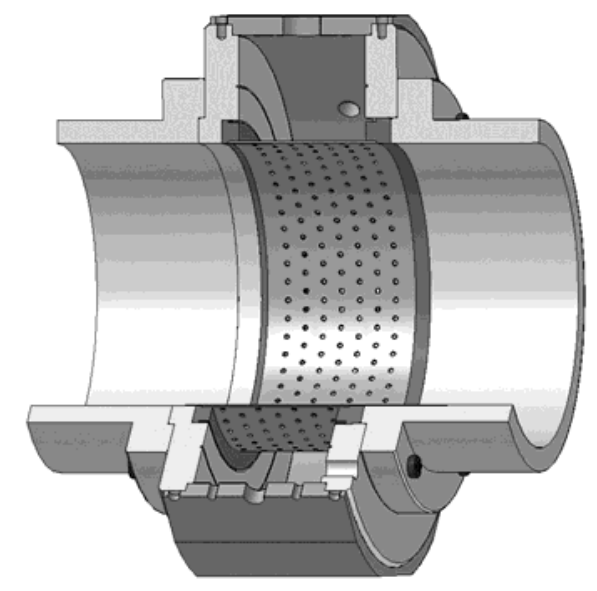

Abbildung 1: Modell eines Bias-Flow Liners zur Dämpfung von Triebwerksschall [Heu10b]

Im Weiteren wird stets die Amplitude der Schallschnelle betrachtet, diese beträgt beispielsweise etwa $70 \mathrm{~mm} / \mathrm{s}$ bei einem Schalldruckpegel von $120 \mathrm{~dB}$ in Luft. Bei den Bias-Flow-Linern ist die Schallschnelle von einer Strömungsgeschwindigkeit überlagert, welche in der Größenordnung von etwa 100 m/s liegt. Um Schallschnelle und Strömungsgeschwindigkeit gleichzeitig zu erfassen, ist eine hohe Dynamik des Messbereichs erforderlich. Zur Bestimmung der Schallschnelleamplitude für Schallfrequenzen im Hörbereich (bis ca. $19 \mathrm{kHz}$ ) werden Geschwindigkeitswerte mit hoher Messrate benötigt. Weiterhin ist eine Messunsicherheit von ca. $1 \mathrm{~mm} / \mathrm{s}$ anzustreben. Ferner wird eine simultane Mehrpunktmessung der Schallschnelle gefordert. Hierfür bieten sich optische Verfahren aus der Strömungsmesstechnik an. Erste Schallschnellemessungen wurden bereits 1976 mit einem Laser-Doppler-Anemometer (LDA) durchgeführt, dabei wurden Schallschnellen bis minimal 0,5 mm/s aufgelöst [Tay76]. Jedoch handelte es sich nur um eine punktförmige Messung. Eine bildgebende Schallschnellemessung erlaubt die Particle Image Velocimetry (PIV), was u. a. von [Han97] bei einer Schallschnelle von minimal $2 \mathrm{~m} / \mathrm{s}$ demonstriert wurde. In [Fis09a] konnten mit PIV sogar Schallschnellen bis $1 \mathrm{~mm} / \mathrm{s}$ aufgelöst werden. Standard-PIVMesssysteme bieten jedoch in der Regel eine zu geringe Messrate, um die Amplitude ohne Unterabtastung zu bestimmen. Dieser Nachteil entfällt bei der Doppler-Global-Velozimetrie (DGV). So wurden bereits in [Fis10] Schallschnellen mit DGV gemessen, jedoch bislang nur als Einpunktmessung mit einer Messdauer von $60 \mathrm{~s}$. 
In diesem Beitrag wird ein DGV-System mit sinusförmiger Frequenzmodulation (FM-DGV) erstmals für die Mehrpunktmessung der Schallschnelle eingesetzt. Im Ergebnis konnte der Schallschnelleverlauf in einem Kundtschen Rohr bestimmt werden. Zudem konnten bei einer Messrate von $50 \mathrm{kHz}$ gleichzeitig mehrere Schallfrequenzen aufgelöst werden. Bei einer Messdauer von lediglich $1 \mathrm{~s}$ betrug die Messunsicherheit minimal $2 \mathrm{~mm} / \mathrm{s}$. Diese Unsicherheit wird gegenwärtig vom Rauschen bei der Photodetektion dominiert und kann durch längere Mittelung unterdrückt werden. Nach der Erhöhung der Messdauer auf $8 \mathrm{~s}$ wurde sogar eine minimale Unsicherheit von $1 \mathrm{~mm} / \mathrm{s}$ erzielt, wobei diese Unsicherheit dann durch Langzeiteffekte (thermische Drifts) limitiert wird.

\section{Messsystem}

Für die Messungen der Schallschnelle wurde hier das FM-DGV-System genutzt, welches u. a. in [Fis09b] ausführlich beschrieben ist. An dieser Stelle soll daher das Messprinzip nur kurz vorgestellt werden. Anschließend wird auf die besonderen Eigenschaften für die Schallschnellemessung eingegangen. Die Messung der Schallschnelle bzw. allgemein der Geschwindigkeit $\vec{v}$ (Abbildung 2 links) in einem Medium erfolgt optisch, basierend auf dem Dopplereffekt bei der Streuung von Licht an bewegten Partikeln, die der Medium beigefügt werden. Die Partikel folgen dem Medium und nehmen näherungsweise dessen Geschwindigkeit $\vec{v}$ an. Die Partikel werden mit Laserlicht beleuchtet, dessen Frequenz sinusförmig moduliert wird und dessen Mittenfrequenz $f_{\mathrm{c}}$ stabilisiert ist. Das an den Partikeln gestreute Licht weist eine geschwindigkeitsabhängige Dopplerfrequenzverschiebung $f_{\mathrm{D}}$ auf. Das Streulicht wird durch eine Absorptionszelle geführt und das transmittierte Licht anschließend mit einem Photodetektor gemessen. Aufgrund der nichtlinearen Transmissions-Frequenz-Funktion (Abbildung 2 rechts) der Absorptionszelle weist das mit dem Detektor gemessene Intensitätssignal neben der Modulationsfrequenz weitere harmonische Anteile auf. Es wird das Verhältnis von 1. und 2. Harmonischer des Detektorsignals gebildet, was die Querempfindlichkeit zur unbekannten Streulichtleistung eliminiert. Der Wert des Quotienten wird somit nur von der Dopplerfrequenz des Lichts bestimmt und kann, nach erfolgter Kalibrierung, einem Messwert für die Geschwindigkeit zugeordnet werden. Dieser Wert entspricht genau der Geschwindigkeitskomponente in Richtung der Winkelhalbierenden zwischen Einfallsrichtung $\vec{i}$ des Laserlichts und Beobachtungsrichtung $\vec{O}$ des Streulichts. Setzt man drei Lichteinfallsrichtungen bzw. drei Beobachtungsrichtungen ein, können alle drei Komponenten des Geschwindigkeitsvektors bestimmt werden [Fis11]. Durch die Verwendung eines Detektorarrays können zudem simultane Mehrpunktmessungen der Geschwindigkeit bzw. der Schallschnelle realisiert werden.
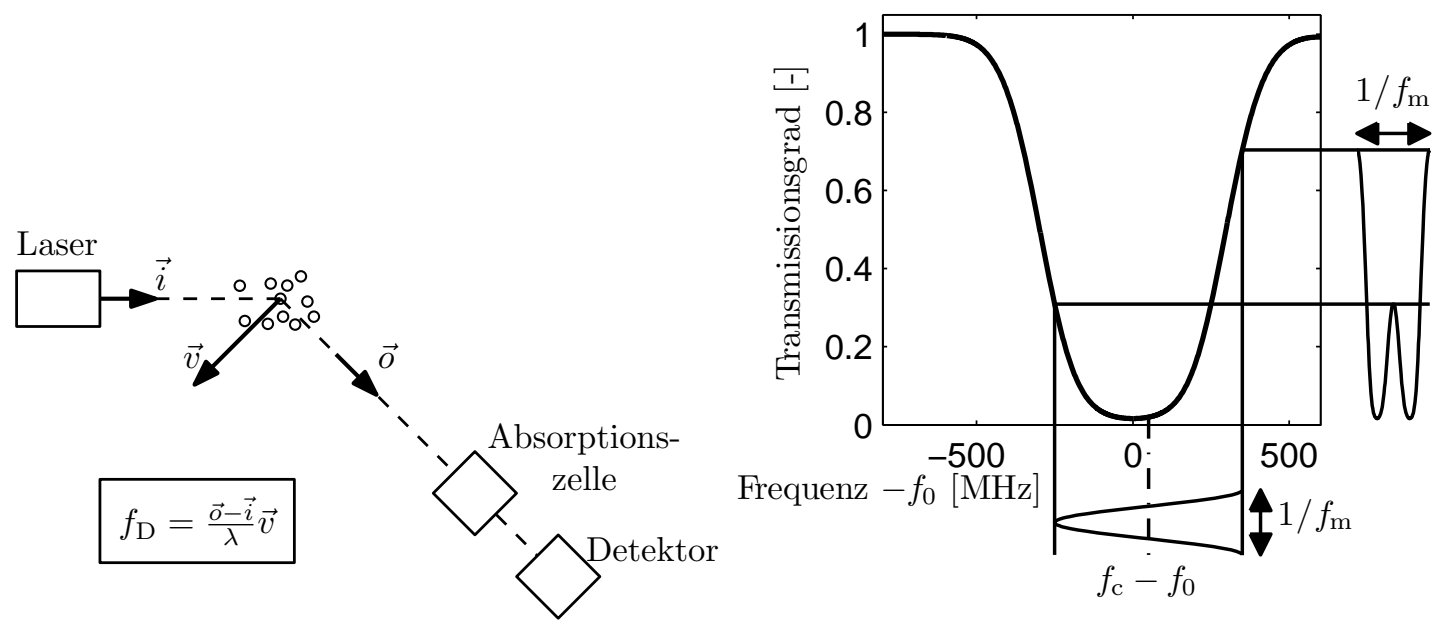

Abbildung 2: Messprinzip FM-DGV (links) sowie Transmissionskennlinie der Absorptionszelle (rechts, Cäsium $\mathrm{D}_{1}$-Linie mit $f_{0}=335 \mathrm{THz}$, Modulationsfrequenz $f_{\mathrm{m}}$ )

Für die im folgenden Abschnitt beschriebenen Schallschnellemessungen wurde ein frequenzmodulierter Diodenlaser (Wellenlänge $\lambda=895 \mathrm{~nm}$ ) mit optischem Verstärker eingesetzt, der eine maximale 
Ausgangsleistung von 600 mW generiert. Hierdurch kann ein hohes Signal-Rausch-Verhältnis und somit eine geringe Messunsicherheit erreicht werden. Bei einer Messdauer von $1 \mathrm{~s}$ beträgt die Unsicherheit aktuell ca. $3 \mathrm{~mm} / \mathrm{s}$. Für eine ausführliche Diskussion des Messunsicherheitsbudgets sei auf [Fis08] verwiesen. Die Messrate beträgt $50 \mathrm{kHz}$, somit ist gemäß dem Nyquist-Shannon-Abtasttheorem eine Auflösung des Geschwindigkeitsspektrums bis maximal 25 kHz möglich, was die Bestimmung der Schallschnellen im Hörbereich gestattet. Die beigefügten Partikel bestehen aus Diethylhexylsebacat, sie folgen der Oszillation der Schallschnelle nahezu schlupffrei (<1\% Schlupf bei $6 \mathrm{kHz}$ [Alb03]). Der Messbereich beträgt $+/-260 \mathrm{~m} / \mathrm{s}$. Für die Mehrpunktmessung wird ein fasergekoppeltes Detektorarray aus 25 Elementen verwendet. Das Messsystem erfüllt damit die im ersten Abschnitt genannten Anforderungen (Mehrpunktmessung, großer Messbereich, hohe Messrate, geringe Messunsicherheit) an die Messung von Schallschnelle und Strömungsgeschwindigkeit in den Triebwerksdämpfern.

\section{Ergebnisse}

Die Mehrpunktmessung der Schallschnelle soll im sogenannten Kundtschen Rohr demonstiert werden. Hierfür wurde ein Plexiglasrohr mit einem Durchmesser von 5,5 cm gewählt. Ein Ende des Rohres wird mit einem starren Kolben aus Aluminium verschlossen. Über die verschiebbare Kolbenposition im Rohr wird die wirksame Rohrlänge $L$ eingestellt. Am gegenüberliegenden, offenen Ende ist eine Schallquelle (Lautsprecher) platziert. Die vom Lautsprecher ausgesandte ebene Schallwelle breitet sich durch das Rohr aus und wird am Kolben reflektiert. Aufgrund der Überlagerung von hin- und rücklaufender Welle bilden sich ein stehendes Schallfeld aus, welches gemessen werden soll. Es sei erwähnt, dass der Schallschnellevektor gemäß der Schallausbreitung einer ebenen Welle nur eine Komponente parallel zur Rohrachse enthält. Der theoretisch zu erwartende Verlauf dieser Schallschnellekomponente ist in Abbildung 3 über der axialen Position im Rohr dargestellt. Dabei sind verschiedene Zeitpunkte eingezeichnet, die den Schwingungsverlauf verdeutlichen sollen. Man erkennt Orte, an denen die Schallschnelleamplitude maximal ist (sogenannte Schwingungsbäuche) und Orte mit einer Schallschnelleamplitude gleich null (Schwingungsknoten).

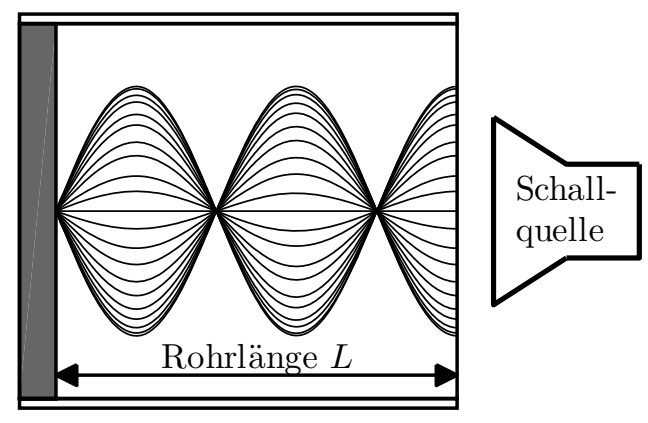

Abbildung 3: theoretischer Schallschnelleverlauf im Kundtschen Rohr für feste Zeitpunkte

Besonders hohe Amplituden der Schallschnelle sind zu erwarten, wenn Resonanz auftritt. Die zugehörige Resonanzbedingung fordert für die Schallfrequenz

$$
f_{\mathrm{s}}=(2 n+1) \frac{C_{\mathrm{s}}}{4 L}, \quad n \in \mathrm{N}
$$

wenn eine bestimmte Rohrlänge (hier: $L \approx 31,8 \mathrm{~cm}$ ) gegeben ist. Dabei bezeichnet $C_{\mathrm{s}} \approx 343 \mathrm{~m} / \mathrm{s}$ die Schallgeschwindigkeit (hier: Luft bei $20^{\circ} \mathrm{C}$ ), also die Ausbreitungsgeschwindigkeit der Schallwelle im Medium. Da in Gleichung (1) beliebige natürliche Zahlen $n$ eingesetzt werden können, können bei fester Rohrlänge prinzipiell mehrere Schallfrequenzen angeregt werden, für die die Resonanzbedingung gleichzeitig erfüllt ist.

Für die Schallschnellemessung mittels FM-DGV im Kundtschen Rohr wurde der Versuchsaufbau gemäß Abbildung 4 gewählt. Die gemessene Geschwindigkeitskomponente (entlang der Winkelhalbierenden von Lichteinfalls- und Beobachtungsrichtung) ist entsprechend des zu erwartenden Schallschnellevektors axial ausgerichtet. 

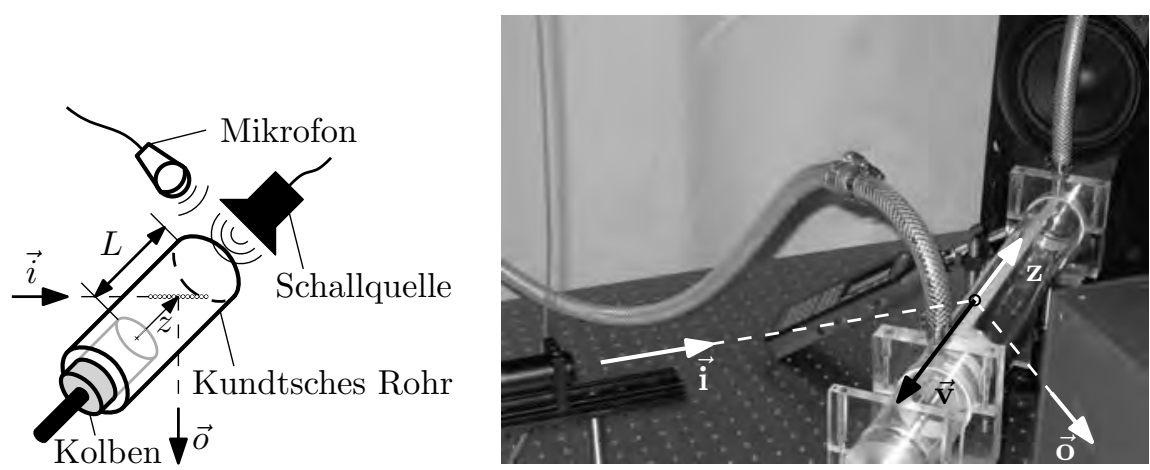

Abbildung 4: Schallschnellemessung mit FM-DGV im Kundtschen Rohr (Skizze und Foto)

Die Geschwindigkeitsmesswerte $v$ wurden für eine Messdauer von $1 \mathrm{~s}$ aufgenommen. Bei der Betrachtung der Schallschnelle sind oft die Geschwindigkeitsamplituden $A_{v}$ von wesentlicher Bedeutung. Das Geschwindigkeitsspektrum erhält man mittels der schnellen Fouriertransformation (FFT) der gemessenen Geschwindigkeitswerte. Ein solches Amplitudenspektrum ist am Beispiel der Schallanregung mit drei Resonanzfrequenzen $8.899 \mathrm{~Hz}, 12.135 \mathrm{~Hz}$ und $16.989 \mathrm{~Hz}$ in Abbildung 5 (links) dargestellt. Man erkennt deutlich die Amplitudenüberhöhungen bei den angeregten Schallfrequenzen. Diese stellen die gesuchten Schallschnelleamplituden dar. Weiterhin ist über dem dargestellten Spektralbereich ein in erster Näherung weißes Rauschen sichtbar, welches vorrangig vom Rauschen bei der Photodetektion herrührt. Im unteren Kilohertz-Bereich zeigen sich zudem überlagerte Frequenzanteile, die auch im Spektrum ohne Schallanregung (Abbildung 5 rechts) vorliegen. Als Ursache hierfür sind die trotz Stabilisierung verbleibenden Regelabweichungen der Lasermittenfrequenzregelung (analoger PID-Regler) zu nennen, welche vom System als Geschwindigkeitsoszillation fehlinterpretiert werden und so zur Verfälschung des Amplitudenspektrums führen. Um diesen Störeffekt zu unterdrücken, sollte in zukünftigen Untersuchungen eine Verringerung der Regelabweichungen durch Optimierung der Lasermittenfrequenzregelung angestrebt werden.
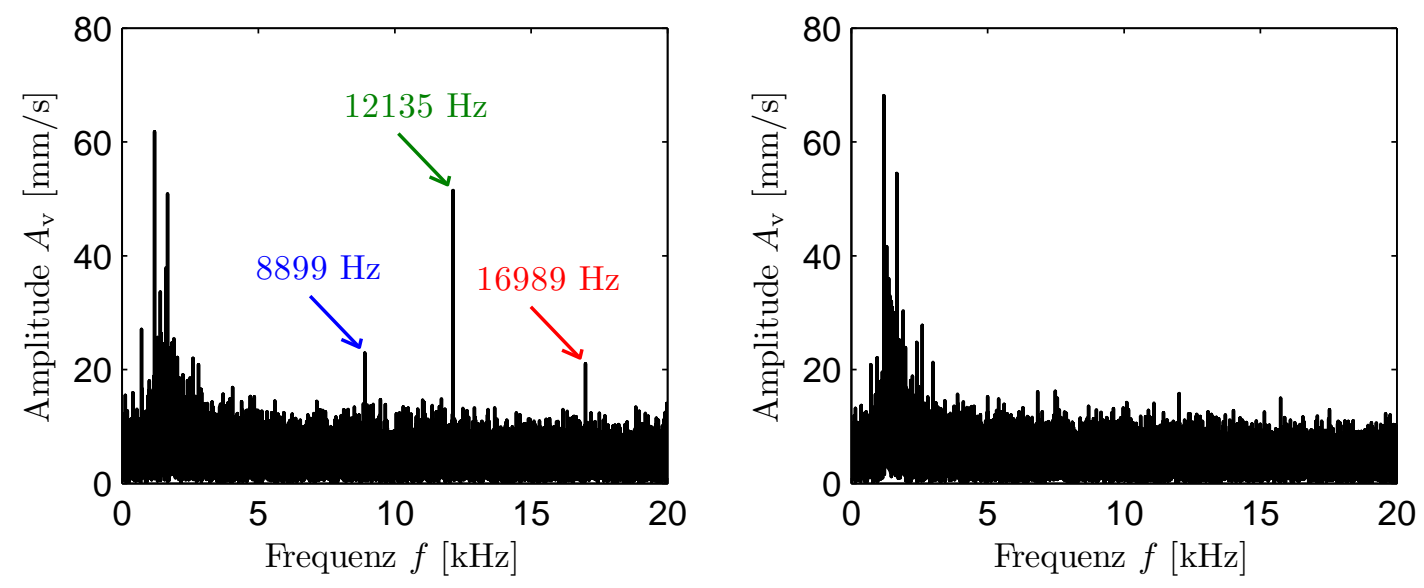

Abbildung 5: beispielhaftes gemessenes Spektrum der Geschwindigkeit mit und ohne Schallanregung

Zunächst soll die minimal messbare Schallschnelle charakterisiert werden. Hierfür wurde die Resonanzfrequenz $f_{\mathrm{s}}=807 \mathrm{~Hz}$ zur Schallanregung gewählt und die Lautstärke variiert. Die Schätzung der Schallschnelleamplituden $\hat{A}_{v}$ wurde mittels Fouriertransformation der Geschwindigkeitsmesswerte beispielhaft für einen Kanals realisiert. In einem ersten Schritt wurde der Betrag des komplexen Fourierkoeffizienten genutzt, d. h. die Auswertung erfolgte ohne Phasenbezug zur Schallanregung. Aus je zehn Amplituden wurde der Mittelwert und dessen Konfidenzintervall für das 95\%-Niveau gebildet. Das Ergebnis ist in Abbildung 6 über dem ebenfalls gemessenen Schalldruckpegel aufgetragen. 


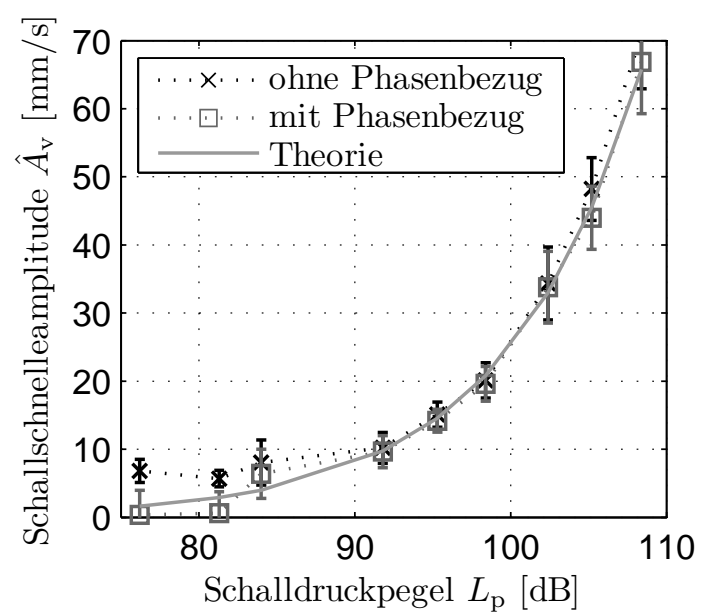

Abbildung 6: gemessene Schallschnelleamplituden bei $f_{\mathrm{s}}=807 \mathrm{~Hz}$ und theoretische Abschätzung Im Vergleich dazu sind die über den theoretischen Zusammenhang (nach [Mös07])

$$
\hat{A}_{\mathrm{v}, \text { Theorie }}=k \frac{\sqrt{2} p_{0} 10^{\frac{L_{\mathrm{p}}}{20 \mathrm{~dB}}}}{Z}
$$

berechneten Amplituden abgebildet. Die hierfür einzusetzenden Größen sind der Schalldruckpegel $L_{\mathrm{p}}$, die sogenannte Schallkennimpedanz $Z \approx 414 \mathrm{Ns} / \mathrm{m}^{3}$ (für Luft bei $20^{\circ} \mathrm{C}$ ) und der genormte Bezugsdruck $p_{0}=2 \cdot 10^{-5} \mathrm{~Pa}$. Da der Schalldruckpegel aus technischen Gründen außerhalb des Rohres gemessen wurde, war eine Skalierung der so berechneten Amplituden mit dem empirisch ermittelten Faktor $k=3,66$ notwendig.

Im Ergebnis treten für kleine Schalldruckpegel (im Bereich kleiner $80 \mathrm{~dB}$ ) systematische Messabweichungen der Schallschnelleamplituden bis maximal $5 \mathrm{~mm} / \mathrm{s}$ auf. Die zufälligen Abweichungen (Standardabweichungen) betragen im Mittel $5 \mathrm{~mm} / \mathrm{s}$. Beide Abweichungen resultieren aus dem verrauschten Geschwindigkeitssignal, welches vor allem durch thermisches Rauschen und Schrotrauschen bei der Photodetektion gestört wird. Die Amplitudenschätzung ist hier gemäß [Sch11] nicht erwartungstreu, da die Phasenlage der Schallanregung nicht berücksichtigt wurde. Der Erwartungswert der Amplitudenschätzung lautet dann

$$
\mathrm{E}\left[\hat{A}_{\mathrm{v}}\right]=\int_{0}^{\infty} \int_{0}^{2 \pi} \hat{a}_{\mathrm{v}}^{2} \frac{\exp \left(-\frac{\hat{a}_{\mathrm{v}}^{2}-2 \hat{a}_{\mathrm{v}} A_{\mathrm{v}} \cos \varphi+A_{\mathrm{v}}^{2}}{2 \operatorname{Var}\left[\hat{A}_{\mathrm{v}}\right]}\right)}{2 \pi \operatorname{Var}\left[\hat{A}_{\mathrm{v}}\right]} \mathrm{d} \varphi \mathrm{d} \hat{a}_{\mathrm{v}}
$$

und kann aus der Varianz $\operatorname{Var}\left[\hat{A}_{\mathrm{v}}\right]$ der geschätzten Amplituden sowie der tatsächlich vorliegenden Amplitude $A_{\mathrm{v}}$ berechnet werden. Für das vorliegende Beispiel wurde dieser Erwartungswert aus Gleichung (3) numerisch berechnet, die Zuordnung der tatsächlichen Amplituden zu den gemessenen Schalldruckpegeln erfolgte wieder gemäß Gleichung (2). Das Ergebnis für den berechneten Erwartungswert ist in Abbildung 7 dargestellt. Zum Vergleich ist jeweils der Mittelwert der gemessenen Amplitude abgebildet. Hierbei zeigt sich eine sehr gute Übereinstimmung. Aufgrund des eineindeutigen Funktionsverlauf kann so zukünftig eine Korrektur der Amplituden und damit eine unverzerrte Schätzung vollzogen werden. 


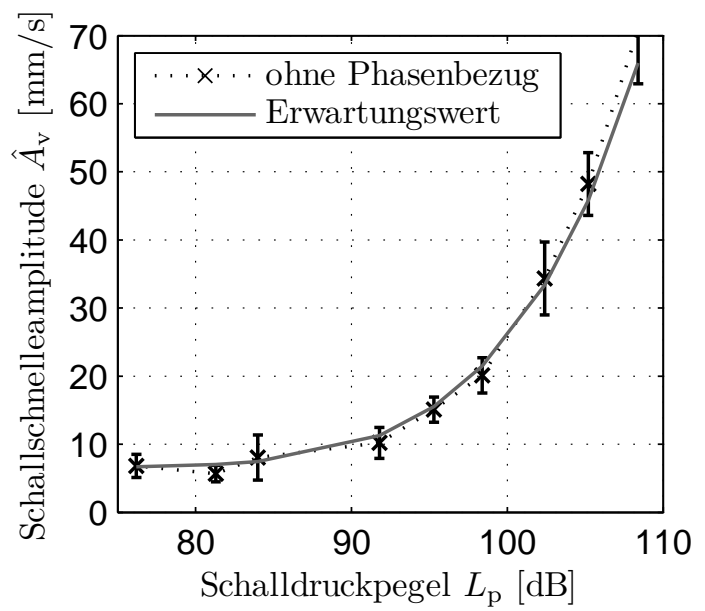

Abbildung 7: Vergleich von gemessener Schallschnelleamplitude (ohne Phasenbezug zur Schallanregung) mit dem Erwartungswert

Erwartungstreue Amplitudenschätzungen sind jedoch auch dann möglich, wenn die Phasenlage der Schallanregung bei der Auswertung berücksichtigt wird. Dazu wurde im zweiten Schritt die Amplitude unter Nutzung des Realteils des komplexen Fourierkoeffizienten gewonnen. Hierfür wird die Phasenlage der gemessenen Schallschnelle in Bezug auf das Anregungssignal benötigt. Diese wurde vorab bei maximaler Schallanregung bestimmt. Diese mit Phasenbezug ermittelten Schallschnelleamplituden sind ebenfalls in Abbildung 6 aufgetragen, in diesem Fall treten keine systematischen Abweichungen auf. Die zufälligen Abweichungen betragen auch hier für alle Messorte im Mittel $5 \mathrm{~mm} / \mathrm{s}$.

Nun folgt die Erweiterung zur simultanen Mehrpunktmessung. Hierfür wurde das fasergekoppelte Detektorarray verwendet. Zudem erfolgte die Schallanregung gleichzeitig mit drei Frequenzen, für die die Resonanzbedingung gemäß Gleichung (1) erfüllt ist. Die Bestimmung der Amplituden erfolgte unter Berücksichtigung der Phasenlage der Schallanregung und ist somit erwartungstreu, wie im vorherigen Absatz diskutiert wurde. Die Messdauer betrug $1 \mathrm{~s}$. Die so erhaltenen Schallschnelleamplituden sind in Abbildung 8 entlang der Position $z$ im Rohr dargestellt. Die Standardabweichung der Amplituden beträgt für alle Messorte im Mittel $4 \mathrm{~mm} / \mathrm{s}$, minimal wurden $2 \mathrm{~mm} / \mathrm{s}$ erzielt. Dies entspricht in etwa den Ergebnissen aus der zuvor beschriebenen Charakterisierung. Ebenfalls in Abbildung 8 eingetragen wurden die theoretischen Verläufe der Schallschnelle. Diese basieren auf der Annahme stehender Wellen für die vorgegebenen Frequenzen. Dabei wurde jeweils die Amplitude im Schwingungsbauch als freier Parameter an die Messwerte angepasst.

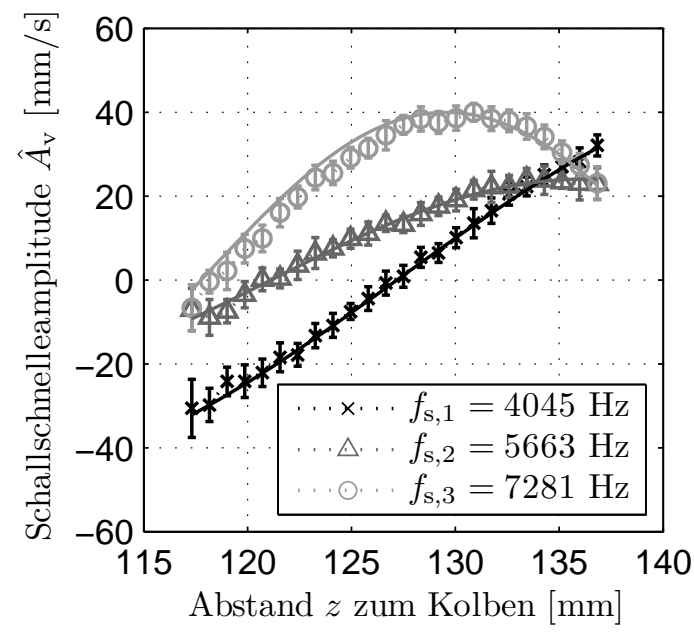

Abbildung 8: gemessener Schallschnelleverlauf im Kundtschen Rohr sowie Theorieabschätzung 
Im Ergebnis zeigt sich eine sehr gute Übereinstimmung zwischen Theorie (durchgezogene Linie) und Experiment (gepunktete Linie) für alle drei Frequenzen: Der theoretische Wert liegt stets im Konfidenzintervall, mit Ausnahme des Schnelleverlaufs für die höchste Frequenz. Diese Abweichungen lassen sich darauf zurückführen, dass mit steigender Frequenz zunehmend weitere Schallausbreitungsmoden zu erwarten sind und somit keine reinen ebenen Wellen mehr vorliegen [Mös07].

Zur weiteren Verringung der Messunsicherheit wurde erstmals eine Schallschnellemessung mit einer ununterbrochenen Messdauer von $8 \mathrm{~s}$ realisiert. Durch die erhöhte Mittelungsdauer wird der Rauscheinfluss stärker unterdrückt, was im Folgenden gezeigt wird. Hierfür wurde eine Mehrpunktmessung für eine beispielhafte Schallfrequenz von $f_{\mathrm{s}}=8.619 \mathrm{~Hz}$ und einer entsprechend angepassten Rohrlänge von $L \approx 41 \mathrm{~cm}$ durchgeführt. Die Schallschnelleamplituden und die dazugehörigen Konfidenzintervalle wurden aus je sechs Wiederholungsmessungen bestimmt und sind in Abbildung 9 dargestellt. Der Vergleich mit der Theorie zeigt auch hier eine gute Übereinstimmung, die Abweichungen des gemessenen Verlaufs von der theoretisch zu erwartenden Sinusform werden wie bereits beschrieben darauf zurückgeführt, dass bei der hier gewählten Anregungsfrequenz die Annahme ebener Schallwellen nur noch eingeschränkt gilt. Ungeachtet dessen wurde eine mittlere

Standardunsicherheit von $2 \mathrm{~mm} / \mathrm{s}$ für alle Messorte erreicht, minimal konnte die eingangs geforderte Messunsicherheit von $1 \mathrm{~mm} / \mathrm{s}$ erzielt werden. Aufgrund der Erhöhung der Messdauer auf $8 \mathrm{~s}$ können jedoch Langzeitdrifteffekte (u. a. thermische Störeinflüsse beim Laser bzw. bei der Absorptionszelle) nicht mehr ausgeschlossen werden, welche nun die Messunsicherheit begrenzen.

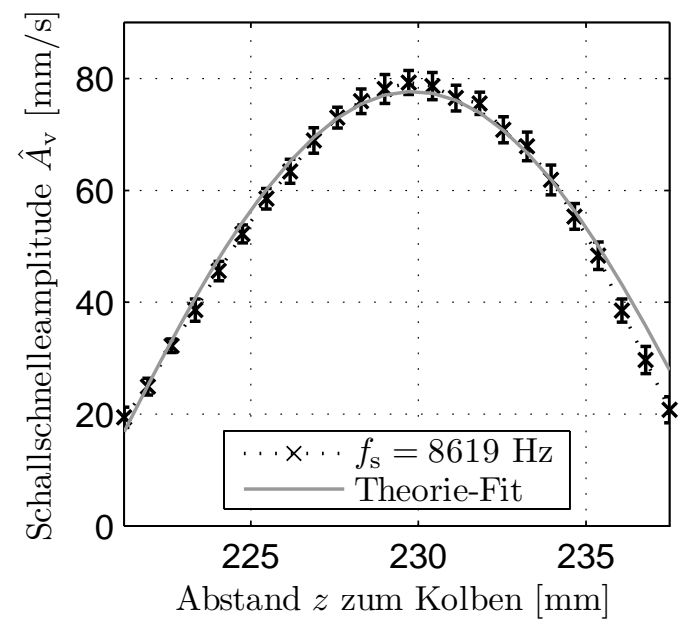

Abbildung 9: Schallschnelleverlauf im Kundtschen Rohr bei $8 \mathrm{~s}$ Messdauer

\section{Fazit}

In diesem Beitrag wurde gezeigt, dass die Schallschnelle berührungslos und simultan an mehreren Punkten mit einem FM-DGV-Messsystem gemessen werden kann. Die Messung erfolgte beispielhaft in einem Kundtschen Rohr. Dabei zeigte sich eine hohe Übereinstimmung mit den theoretisch zu erwartenden Schallschnelleverläufen. Die auftretende Messunsicherheit betrug minimal $2 \mathrm{~mm} / \mathrm{s}$, bei einer Messdauer von lediglich $1 \mathrm{~s}$. Im Ergebnis der erstmaligen Messung mit $8 \mathrm{~s}$ Messdauer konnte sogar eine minimale Messunsicherheit von $1 \mathrm{~mm} / \mathrm{s}$ erzielt werden. Bei einer Messrate von $50 \mathrm{kHz}$ konnten zudem erstmals mehrere Frequenzen im hörbaren Bereich gleichzeitig aufgelöst werden. Mit dem verwendeten Verfahren sind zudem prinzipiell mehrkomponentige Messungen der Schallschnelle möglich, was die Möglichkeit für die berührungslose und daher rückwirkungsfreie Messung im Nahfeld von Schallquellen eröffnet. Da die Messung optisch erfolgt, lässt sie sich zukünftig auch bei hohen Temperaturen, z.B. in Brennkammern von Gasturbinen einsetzen. Die Messtechnik hat somit ein hohes Potential für den Einsatz in Triebwerksschalldämpfern und kann zum Verständnisgewinn der dort auftretenden aeroakustischen Phänomene eingesetzt werden. Zusammenfassend werden damit langfristig Perspektiven für die Optimierung der Liner zur Reduzierung von Triebwerkslärm geschaffen. 


\section{Danksagung}

Die Autoren danken Raimund Schlüßler für die tatkräftige Unterstützung bei der Durchführung der Messungen. Ebenso sei der Deutschen Forschungsgemeinschaft für die Förderung des Projekts Cz55/25-1 gedankt. Weiterhin gilt André Fischer und Dr. Friedrich Bake (Deutsches Zentrum für Luft- und Raumfahrt, Berlin) unser Dank für die wertvollen Anregungen.

\section{Literatur}

[Alb03] H.-E. Albrecht, M. Borys, N. Damaschke und C. Tropea: Laser Doppler and Phase Doppler Measurement Techniques. Springer-Verlag, Berlin, Heidelberg, New York, 2003.

[Eur01] EUROPEAN COMmISSION: European Aeronautics: A Vision for 2020. Office for official Publications of the European Communities, 2001.

[Fis08] A. FISCHER ${ }^{1}$, J. KöNIG und J. CZARSKE: Speckle noise influence on measuring turbulence spectra using time-resolved Doppler global velocimetry with laser frequency modulation. Measurement Science and Technology, 19(12):125402-125413, 2008.

[Fis09a] A. FISCHER ${ }^{2}$, F. BAKE, L. ENGHARDT, I. RÖHLE und S. BuSSE: Acoustic Plane Wave Decomposition by Means of Synchronised PIV. In: 15th AIAA/CEAS Aeroacoustics Conference, No. 2009-3299, Miami, 2009.

[Fis09b] A. FISCHER ${ }^{1}$, L. BütTNer, J. CZARSKE, M. EgGeRT und H. MüLleR: Measurements of velocity spectra using time-resolving Doppler global velocimetry with laser frequency modulation and a detector array. Experiments in Fluids, 47(4):599-611, 2009.

[Fis10] A. FISCHER ${ }^{2}$, A. RAUSCH, F. BAKE und I. RÖHLE: Optische Schallschnellemessung mittels Doppler Global Velocimetry. In: Fortschritte der Akustik DAGA 2010. 36. Deutsche Jahrestagung für Akustik, Berlin, 2010.

[Fis11] A. FISCheR ${ }^{1}$, L. BütTner, J. CZARSke, M. Gottschall, R. MAILACH und K. Vogeler: Investigation of the tip clearance flow in a compressor cascade using a novel laser measurement technique with high temporal resolution. In: ASME Turbo Expo, No. GT2011-45176, Vancouver, 2011.

[Han97] D. B. HANN und C. A. GREATED: The measurement of flow velocity and acoustic particle velocity using particle-image velocimetry. Measurement Science and Technology, 8(12):1517-1522, 1997.

[Heu10a] C. HeuwinkEL, L. ENGHARDt, F. BAKE, S. SAdig und M. GerendÁs: Establishment of a High Quality Database for the Modelling of Perforated Liners. In: ASME Turbo Expo, No. GT2010-22329, Glasgow, 2010.

[Heu10b] C. HeuWinkel, A. Fischer ${ }^{2}$, I. RÖHLE, L. EnghaRdt, F. BAKE, E. PIOT und F. MichelI: Characterization of a Perforated Liner by Acoustic and Optical Measurements. In: 16th AIAA/CEAS Aeroacoustics Conference, No. 2010-3765, Stockholm, 2010.

[Mös07] M. MöSER: Technische Akustik. Springer-Verlag, Berlin, Heidelberg, New York, 2007.

[Sch11] R. SCHLÜßLER, A. FISCHER ${ }^{1}$, L. BÜTTNER und J. CZARSKE: Optische Schallschnellemessung mittels Doppler-Global-Velozimeter mit Laserfrequenzmodulation. In: 19. Fachtagung "Lasermethoden in der Strömungsmesstechnik", Ilmenau, 2011.

[Tay76] K. J. TAYLOR: Absolute measurement of acoustic particle velocity. Journal of the Acoustical Society of America, 59(3):691-694, 1976.

\footnotetext{
${ }^{1}$ Andreas Fischer
}

${ }^{2}$ André Fischer 